\title{
Polymorph selection and nucleation pathway in the crystallization of Hertzian spheres
}

\author{
Wenze Ouyang, ${ }^{1, *}$ Cuiliu Fu, ${ }^{2}$ Zhiwei Sun, ${ }^{1}$ and Shenghua $\mathrm{Xu}^{1,3, \dagger}$ \\ ${ }^{1}$ Key Laboratory of Microgravity (National Microgravity Laboratory), Institute of Mechanics, Chinese Academy of Sciences, \\ Beijing 100190, China \\ ${ }^{2}$ State Key Laboratory of Polymer Physics and Chemistry, Changchun Institute of Applied Chemistry, Chinese Academy of Sciences, \\ Changchun 130022, China \\ ${ }^{3}$ School of Engineering Science, University of Chinese Academy of Sciences, Beijing 100049, China \\ (Received 5 May 2016; revised manuscript received 26 June 2016; published 17 October 2016)
}

\begin{abstract}
The crystallization process of Hertzian spheres is studied by means of molecular dynamics simulations in an $N P T$ ensemble where the total number of particles $N$, the pressure $P$, and the temperature $T$ are kept constant. It has been observed that the bond orientational ordering rather than the translational ordering (density) plays a primary role. The crystal polymorphs are determined by the state points. Under the conditions of small supercooling, the system is likely to be nucleated into crystals that have a preference for the metastable bcc structure, which can be regarded as a manifestation of the Alexander-McTague mechanism. In contrast, small nuclei are found to have a preference for fcc symmetry under conditions of a high degree of supercooling. Prestructured precursors that act as seeds and wet on the nuclei during nucleation always have a high degree of bcc-like ordering, despite different state points. The results above may provide a clue to the understanding of the crystallization process in core-softened particles.
\end{abstract}

DOI: 10.1103/PhysRevE.94.042805

\section{INTRODUCTION}

Crystallization, as an important process in which a structural ordering phase is formed from a disordered state, has attracted a lot of interest in the field of soft matter physics and material science. Due to its complexity and subtle details, the understanding of the crystallization process is not yet complete and the underlying mechanism is still being debated.

In order to explain the observations and to discover the mechanism in crystallization, some different criteria have been provided. Classical nucleation theory (CNT) presents a simple picture in which the nucleus is formed from a initial uniform liquid. Assuming a free-energy barrier to cross, the nucleus can only grow when it exceeds a critical size. So nucleation is a one-step process in the CNT framework, where the only key players are an initial disordered liquid and final ordered solid phase. In 1897, Ostwald formulated the most credited criterion, which was named Ostwald's step rule, stating that the crystal phase nucleated from the liquid should not be the one that is thermodynamically most stable, but the one that is closest in free energy to the liquid phase [1]. Alexander and McTague proposed a criterion different from Ostwald's step rule. In three dimensions and with a simple fluid where a weak first-order phase transition occurs, they said the body-centered-cubic (bcc) phase should be uniquely favored in the early stages of crystallization [2]. Later experiments on rapidly cooled metal melts [3], block copolymer solutions [4], and charged colloidal suspensions [5], which had a stable face-centered-cubic (fcc) phase, showed the nucleation of a metastable bcc phase. ten Wolde et al. reported a numerical simulation study of crystal nucleation in a Lennard-Jones system at moderate supercooling [6,7]. The precritical nucleus was found to be predominantly bcc ordered, despite the

\footnotetext{
*oywz@imech.ac.cn

†xush@imech.ac.cn
}

stable structure being a face-centered-cubic (fcc) crystal. As the nucleus grew to its critical size, the core became fcc ordered while its interface retained a high degree of bcc-like ordering. Desgranges and Delhommelle performed a more detailed study on the polymorphism of the Lennard-Jones system, showing that the internal structure of the precritical, critical, or postcritical nucleus could be controlled by tuning the pressure [8]. In the review article, Delhommelle continued to show that one might achieve control of polymorphism in a variety of systems by modifying the thermodynamic conditions of crystallization [9]. Recently, it was further suggested that crystal nucleation was a discrete two-step process, in which the first step involved the formation of preordered precursors with a relatively dense or relatively ordered liquid structure [10-15]. Valeriani and co-workers found that the crystal nucleation of hard spheres from a metastable suspension could be considered to occur via a multistep mechanism, with the number of steps depending on the number of cutoffs monitored [16]. However, Tanaka and co-workers pointed out that the process of crystal nucleation was not discrete but continuous at the microscopic level, and that local breakdown of rotational symmetry (or bond orientational ordering), rather than the density fluctuations, played a major role in the nucleation stage [17-19]. As for the precursors, in some cases, they can sometimes be less dense than the liquid they form from $[20,21]$.

So far, most studies of crystallization have focused on systems with hard divergent repulsions at a short distance. These systems include the simplest and well-known hard spheres $[14,17,18]$, the Lennard-Jones model [6-8], chargestabilized colloids which have repulsive Yukawa interactions [5,22-25], etc. On the contrary, there are some systems consisting of soft particles whose interactions are even core softened, e.g., emulsions, soft colloids, microgels, many macromolecules, and their self-assembled entities. It has been established that the phase behaviors, especially the solidification of soft particles, are qualitatively different from those of hard ones [26-29], as the shape of the repulsive 
potential determines the phase behaviors of a system [30]. Here, one may consider an extreme class of repulsive potentials that are bounded, i.e., they remain finite for the whole range of interparticle separations, even with full overlap between the particles. Many previous works have suggested that systems with a bounded potential have rich and complex phase behaviors. For instance, Likos et al. derived a simple criterion to determine whether a system of bounded potentials showed reentrant melting or clustering [26]: Reentrant melting occurred for bounded potentials with a positive definite Fourier transform. Otherwise, clustering and freezing were expected to occur at all temperatures. As the solidification phase diagram of soft particles is so different from that of hard ones, an open and interesting question arises as to whether the criteria of crystallization occurring in systems with hard divergent repulsions are still valid in a system of soft particles.

To the best of our knowledge, there are very few studies on the crystallization process and corresponding mechanisms in a system with a bounded potential [31-34]. For instance, Mithen et al. [34] very recently studied the crystallization of the Gaussian core model (GCM) to find that the nuclei formed were typically "mixed" and dependent on the state point of the GCM phase diagram, which is consistent with the observations of Russo and Tanaka [32]. Among those systems with a bounded potential, the Hertz potential is another model which describes the change in the elastic energy of two deformable objects when subjected to an axial compression. Recently, this model has also been used in a number of experimental studies to represent the interaction of deformable soft colloids [35-38]. In the present work, we will take advantage of a previously constructed phase diagram [28] to investigate the crystallization process so as to address homogeneous nucleation in Hertzian spheres. Although the phase diagram of Hertzian spheres exhibits a complex phase behavior and a succession of Bravais crystals, especially at very low temperature [28], we only concentrate on the regions of fcc and bcc phases which have attracted much interest in computational, theoretical, and experimental investigations. Via molecular dynamics (MD) simulations in an NPT ensemble where the total number of particles $N$, the pressure $P$, and the temperature $T$ are kept constant, we will study the local packing symmetry to observe the crystal polymorph and the nucleation pathway.

\section{MODEL AND SIMULATION METHODOLOGY}

Soft spherical particles interact via the Hertz potential, which is given by

$$
U\left(r_{i j}\right)= \begin{cases}\epsilon\left(1-r_{i j} / \sigma\right)^{5 / 2}, & r_{i j}<\sigma, \\ 0, & r_{i j} \geqslant \sigma,\end{cases}
$$

where $r_{i j}$ is the pair distance between the centers of the $i$ th particle and the $j$ th particle. The parameters $\epsilon$ and $\sigma$ govern the strength and maximum distance of the interaction, respectively. For Hertzian spheres, the maximum distance of the interaction $\sigma$ is equal to their diameters.

To investigate the crystallization process of Hertzian spheres, MD simulations are performed in an NPT ensemble. For convenience, the reduced units are used in the simulation. The basic units are chosen as follows: energy unit $\epsilon$, length unit $\sigma$, and the mass of particle $m$. Periodic boundary conditions are applied. The equation of motion is integrated using the velocity Verlet algorithm $[39,40]$ with a time step $\delta t=0.05$. The constant temperature and constant pressure are controlled via a Berendsen thermostat and barostat [41].

During the study of the crystallization process, we need to identify crystal particles. Here, we use the bond orientational order parameters that were originally introduced by Steinhardt et al. [42] and applied by Frenkel and co-workers [6,7] to distinguish the crystal particles from the liquid particles. First, we consider a set of nearest neighbors of a particle $i$ as all particles $j$ that are within a given radius $r_{q}$ from $i$. The value of $r_{q}$ is chosen to be close to the first minimum of the radial distribution function (RDF). Then, the complex vector $q_{l m}(i)$ of particle $i$ is defined by

$$
q_{l m}(i)=\frac{1}{N_{n b}(i)} \sum_{j=1}^{N_{n b}(i)} Y_{l m}\left(\mathbf{r}_{i j}\right) .
$$

The sum runs over all nearest neighbors of particle $i$. The functions $Y_{l m}\left(\mathbf{r}_{i j}\right)$ are the spherical harmonics, $l$ is a freeinteger parameter, and $m$ is an integer that runs from $m=-l$ to $m=l$. To identify solid particles, the integer parameter $l$ is taken as $l=6$ and $q_{6 m}(i)$ should be normalized,

$$
d_{6 m}(i)=\frac{q_{6 m}(i)}{\left[\sum_{m=-6}^{6}\left|q_{6 m}(i)\right|^{2}\right]^{1 / 2}} .
$$

Using the normalized complex vectors $d_{6 m}(i)$, a scalar product which measures the correlation between neighboring particles $i$ and $j$ is defined by

$$
S_{i j}=\sum_{m=-6}^{6} d_{6 m}(i) \cdot d_{6 m}^{*}(j),
$$

where the superscript $*$ indicates complex conjugation. Two neighboring particles $i$ and $j$ are considered to be connected if $S_{i j}$ exceeds a given value, typically $S_{i j}>0.7$. A particle will be identified as solid if the number of its connected neighbors $\zeta$ is above a certain threshold, typically 8 .

For a further structural identification of each crystal particle, we use the coarse-grained bond order parameters introduced by Lechner and Dellago [43]. Different from Eq. (2), the averaged complex vector $\bar{q}_{l m}(i)$ of particle $i$ is defined by

$$
\bar{q}_{l m}(i)=\frac{1}{N_{n b}(i)} \sum_{k=0}^{N_{n b}(i)} q_{l m}(k) .
$$

The sum above from $k=0$ to $N_{n b}(i)$ runs over all neighbors of particle $i$ plus the particle $i$ itself. Then, we construct the quantities of the averaged version,

$$
Q_{l}(i)=\left(\frac{4 \pi}{2 l+1} \sum_{m=-l}^{l}\left|\bar{q}_{l m}(i)\right|^{2}\right)^{1 / 2}
$$

and

$$
\begin{aligned}
W_{l}(i)= & \sum_{m_{1}+m_{2}+m_{3}=0}\left(\begin{array}{ccc}
l & l & l \\
m_{1} & m_{2} & m_{3}
\end{array}\right) \\
& \times \frac{\bar{q}_{l m_{1}}(i) \bar{q}_{l m_{2}}(i) \bar{q}_{l m_{3}}(i)}{\left(\sum_{m=-l}^{l}\left|\bar{q}_{l m}(i)\right|^{2}\right)^{3 / 2}}
\end{aligned}
$$


where the term in parentheses is the Wigner $3-j$ symbol. The integers $m_{1}, m_{2}$, and $m_{3}$ run from $-l$ to $l$, but only combinations with $m_{1}+m_{2}+m_{3}=0$ are allowed. To further identify the solidlike particles, we take advantage of the different symmetries that the crystals have on the $W_{6}$ and $W_{4}$ axis [32]. The following criterion for crystal classification is used: Crystal particles are first identified using the method presented by Frenkel and co-workers [6,7]. Then, a crystal particle $i$ will be considered to be (i) fcc as $W_{6}(i)<0$ and $W_{4}(i) \leqslant 0$, (ii) hexagonal-close-packed (hcp) as $W_{6}(i)<0$ and $W_{4}(i)>0$, and (iii) bec as $W_{6}(i) \geqslant 0$.

To compare the stability of different solid phases (fcc, hcp, bcc), we employ the thermodynamic integration technique to calculate the free energy for the crystal structures [40]. The basic idea of this method is to connect continuously the solid state under consideration (system 1 with potential energy $U_{1}$ ) to a reference solid (system 0 with potential energy $U_{0}$ ) whose free energy can be calculated exactly. For the reference solid, we choose an Einstein crystal in which the noninteracting particles are coupled to their lattice sites by harmonic springs. So, a hybrid system is sampled with potential $U_{\lambda}=U_{0}+$ $\lambda\left(U_{1}-U_{0}\right)$, where $\lambda$ is the switching parameter and can vary between 0 and 1 . Then, the Helmholtz free energy of system 1 is obtained through the formula

$$
F=F_{0}+\int_{0}^{1} d \lambda\left\langle U_{1}-U_{0}\right\rangle_{\lambda}
$$

The free energy of the Einstein crystal $F_{0}$ is given exactly by

$$
F_{0}=-\frac{3 N}{2 \beta} \ln \left(\frac{2 \pi}{\alpha \beta}\right),
$$

where $\beta=1 / k_{B} T . \alpha$ is the spring constant of the Einstein crystal, which is chosen to make the mean-squared displacements for $\lambda=0$ and $\lambda=1$ be equal,

$$
\alpha=\frac{3}{\beta\left\langle\Delta R^{2}\right\rangle_{\lambda=1}} .
$$

In order to avoid the problem that the integrand in Eq. (8) diverges for $\lambda=1$, simulations of $U_{\lambda}$ should be performed under the constraint of a fixed center of mass. In practice, we usually calculate the excess free energy $F_{\text {ex }}=F-F_{\text {id }}$, where $F_{\text {id }}$ is the free energy of the ideal gas. Taking into account the corrections of constraints and finite size effects, we can get

$$
\begin{aligned}
\beta F_{\mathrm{ex}}= & \beta F_{0}+\beta \int_{0}^{1} d \lambda\left\langle U_{1}-U_{0}\right\rangle_{\lambda}^{\mathrm{CM}}+(1-N) \ln \rho \\
& -\frac{3}{2} \ln \left(\frac{\beta \alpha m}{2 \pi}\right)-2 \ln N+N-\frac{1}{2} \ln 2 \pi .
\end{aligned}
$$

During the thermodynamic integration, we slowly transform the candidate crystal into an Einstein crystal by changing the switching parameter from 1 to 0 with an interval of $\Delta \lambda=0.01$ to ensure that the accuracy of the calculation is satisfactory. In a simulation with a fixed center of mass, particles can undergo only limited excursions along the run. Therefore, the periodic boundary conditions are not used for the motion of each particle and a particle that happens to move out of the simulation box does not need to be put back.

\section{RESULTS AND DISCUSSION}

According to a previously constructed phase diagram [28], Hertzian spheres can be crystallized below a melting temperature $T_{m} \approx 8.84 \times 10^{-3}$. Here, we mainly concentrate on two typical temperatures which represent different supercooling: One is at $T=5 \times 10^{-3}$ and the other is far below the melting temperature at $T=2.5 \times 10^{-3}$. To follow the crystallization process of the system, the number of particles is typically chosen to be $N=4000$. The particles are initially placed on random positions and their velocities satisfy the MaxwellBoltzmann distribution with the temperature above the melting point. After a relaxation of $10^{5} \delta t$, the system is quenched to the desired temperature and $N P T$ simulations are carried out long enough until the crystallization is completed. Most of the simulations run over a total of $2 \times 10^{6} \delta t$ and the last $10^{5} \delta t$ are used to calculate the average values of the parameters needed.

In order to examine the validity of Ostwald's step rule in the crystallization process of Hertzian spheres, we have calculated the free energy at each state point studied in this work. The results of the free-energy calculations are presented in Table I. If Ostwald's step rule applies to the crystallization of Hertzian

TABLE I. Excess Helmholtz free energy per particle for fcc, hcp, and bec crystals under different thermodynamic conditions ( $P$ and $T$ ) considered in the study. The thermodynamically stable phase for each state point shown in the table is according to the phase diagram of Ref. [28]. The free energy of different crystals is calculated with the Einstein crystal method. The simulations of the Einstein crystal are performed in the canonical $(N V T)$ ensemble with $N=4000$ for fcc and hcp crystals, and with $N=4394$ for bcc crystals. In each

\begin{tabular}{|c|c|c|c|c|c|}
\hline$P$ & $\rho$ & Stable phase & $\beta F_{\mathrm{fcc}}^{\mathrm{ex}}$ & $\beta F_{\mathrm{hcp}}^{\mathrm{ex}}$ & $\beta F_{\mathrm{bcc}}^{\mathrm{ex}}$ \\
\hline \multicolumn{6}{|c|}{$T=5.0 \times 10^{-3}$} \\
\hline 0.25 & 1.872 & $\mathrm{fcc}$ & $9.979(3)$ & $9.986(3)$ & $10.138(3)$ \\
\hline 0.3 & 1.954 & fcc & $11.187(2)$ & $11.184(3)$ & $11.316(3)$ \\
\hline 0.4 & 2.111 & fcc & $13.831(2)$ & $13.830(3)$ & $13.862(3)$ \\
\hline 0.45 & 2.114 & bcc & $13.878(2)$ & $13.883(2)$ & $13.912(3)$ \\
\hline 0.5 & 2.25 & bcc & $16.495(3)$ & $16.505(2)$ & $16.415(2)$ \\
\hline 0.6 & 2.373 & bcc & $19.086(2)$ & $19.105(3)$ & $18.897(2)$ \\
\hline 0.7 & 2.493 & bcc & $21.695(6)$ & 21.64(1) & $21.486(2)$ \\
\hline 0.8 & 2.604 & bcc & $24.252(9)$ & $24.16(1)$ & $24.015(3)$ \\
\hline 0.9 & 2.712 & bcc & $26.78(1)$ & $26.73(1)$ & $26.563(2)$ \\
\hline 1.0 & 2.816 & bcc & $29.34(1)$ & $28.92(1)$ & $29.133(2)$ \\
\hline \multicolumn{6}{|c|}{$T=2.5 \times 10^{-3}$} \\
\hline 0.1 & 1.607 & $\mathrm{fcc}$ & $8.664(2)$ & $8.663(2)$ & $8.92(2)$ \\
\hline 0.2 & 1.814 & fcc & $12.541(3)$ & $12.532(2)$ & 12.91(2) \\
\hline 0.3 & 1.966 & fcc & $16.709(2)$ & $16.696(2)$ & $17.05(2)$ \\
\hline 0.4 & 2.102 & fcc & $21.292(2)$ & $21.261(2)$ & $21.568(2)$ \\
\hline 0.45 & 2.172 & fcc & $23.922(3)$ & $23.894(2)$ & $24.018(2)$ \\
\hline 0.5 & 2.266 & bcc & $27.648(2)$ & $27.638(2)$ & $27.483(2)$ \\
\hline 0.6 & 2.372 & bcc & $32.194(3)$ & $32.192(3)$ & $31.737(2)$ \\
\hline 0.7 & 2.5 & bcc & $37.83(1)$ & $37.76(2)$ & $37.211(2)$ \\
\hline 0.8 & 2.608 & bcc & $42.84(2)$ & $42.65(2)$ & $42.148(2)$ \\
\hline 0.9 & 2.715 & bcc & $48.00(2)$ & $47.70(2)$ & $47.273(2)$ \\
\hline 1.0 & 2.814 & bcc & $52.89(3)$ & $52.60(2)$ & $52.162(2)$ \\
\hline
\end{tabular}
simulation, the equilibration and the production runs are $10^{5}$ time steps. The thermodynamic integration [see Eq. (11)] is done with an interval of $\Delta \lambda=0.01$. The last digit in parentheses indicates the statistical error. 


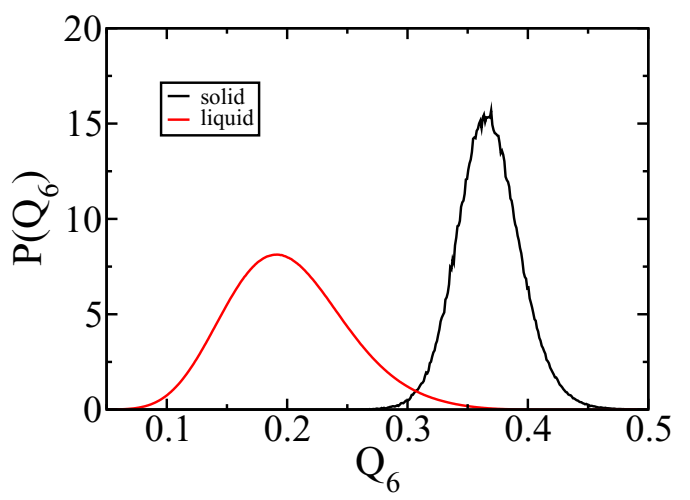

FIG. 1. Probability distribution of $Q_{6}$ for liquid and solid particles in metastable fluid. The state point is $T=5 \times 10^{-3}$ and $P=0.25$. The black line represents the curve for solid particles and the red line represents the curve for liquid particles.

spheres, we may expect that the crystal structure nucleated first should be the one with the highest $F_{\text {ex }}$ whose free energy is closest to the metastable liquid phase. However, this does not always happen because the system shows different nucleation behaviors for different state points. In the following, we will show our observations of the crystallization process at different state points. Notice here that we have performed 100 independent NPT simulations for each state point to eliminate the effect of the initial conditions and to get better statistics.

\section{A. Metastable liquid and precursors}

For a higher temperature $T=5 \times 10^{-3}$, the system will exhibit a reentrance of the fluid phase according to its phase diagram [28]: One may see a sequence of fluid, solid, and again a fluid phase upon increasing the pressure. Here, we only focus on the region of the fcc and bcc phase. The thermodynamically stable solid phase is fcc at low pressures, while the thermodynamically stable solid phase is bcc at high pressures (see Table I). Based on the phase diagram of Hertzian spheres [28], it is known that the pressure of the boundary of a liquid-fcc phase transition is about $P_{\mathrm{LS}}=0.12$. We first perform the simulations at the state point $T=5 \times 10^{-3}$ and $P=0.25$ where fcc is thermodynamically stable. Actually, we have also used some other pressures lower than $P=0.25$ when $T=5 \times 10^{-3}$, but do not see the emergence of crystallization, probably due to the extremely long time of the nucleation event which exceeds the maximum time steps of simulations. When $P=0.25$ and $T=5 \times 10^{-3}$, we speculate that the system, in most of simulations, is not only eventually crystallized, but also attains a steady-state metastable stage which is in the fluid phase and exists for a rather long time.

To distinguish crystal particles from liquid particles, we can use the method proposed by Frenkel and co-workers [6,7]. However, it is also necessary to use some other bond orientational order parameters for a better analysis of both crystal and liquid particles. A coarse-grained bond orientational order parameter $Q_{6}$ [see Eq. (6)] is a good choice to follow the liquid-solid phase transition. Figure 1 displays the curves of $Q_{6}$ probability distribution for liquid and crystal particles separately in the metastable supercooled fluid when $T=$ $5 \times 10^{-3}$ and $P=0.25$. Obviously, solid particles have a higher $Q_{6}$ than liquid particles and the overlap between their curves of the $Q_{6}$ probability distribution is small. We also monitor the liquid particles that transform into crystals and find those particles always have high $Q_{6}$. So this means that the order parameter $Q_{6}$ plays an important role in nucleation. The preordered liquid particles with high $Q_{6}$ can be considered to be mediated precursors, as the twostep and continuous scenario suggests [19]. Of course, such precursors not only appear in the metastable stage but also exist in the crystallization stage. In other words, precursors as noncrystalline clusters with a pronounced short-range order are first formed and then the crystal nucleus appears inside the precursors. In such a picture, crystal nucleation can be understood as the conversion of precursors into crystals.

We have seen that the development of the coarse-grained bond order parameter $Q_{6}$ drives the whole crystallization process, as is also found in other systems $[17,18,32]$. Therefore, the precursors can be identified by $Q_{6}$ alone (we typically define as $Q_{6} \geqslant 0.3$ ). Here, some may ask why an original Steinhardt bond order parameter $q_{6}$ is not used. Indeed, the development of $q_{6}$ can also drive the crystallization, but the overlap between the $q_{6}$ probability distribution of crystal particles and that of liquid particles is significant. The other disadvantage of using $q_{6}$ is that high $q_{6}$ is a representation of icosahedral particles which has been revealed in both experiments and simulations to act as an inhibitor to crystallization [44]. By means of the method presented in Ref. [45], we identify the particles in an icosahedral environment so as to find that icosahedral particles always have high $q_{6}$.

It is interesting to study the structure of the relatively ordered precursors, as the precursors act as the seeds of crystal nucleation. In Sec. II, different crystal particles can be identified by their different symmetries on the $W_{6}-W_{4}$ plane. Here, we exploit these symmetries to characterize the different structures of the precursors. A liquid particle will be considered to belong to the precursors if its local bond order parameter $Q_{6}$ is larger than a threshold $Q_{6}^{\text {thr }}$, then it is (i) fcc-like as $W_{6}<0$ and $W_{4} \leqslant 0$, (ii) hcp-like as $W_{6}<0$ and $W_{4}>0$, and (iii) bcc-like as $W_{6} \geqslant 0$. Using different $Q_{6}^{\text {thr }}$ thresholds, we calculate the probability distribution of $W_{4}$ for the liquid particles of a metastable fluid with the character of $W_{6}<0$ and $Q_{6} \geqslant Q_{6}^{\text {thr }}$. As is shown in the top panel of Fig. $2, W_{4} \leqslant 0$ means a particle is in a fcc-like environment and $W_{4}>0$ means a particle is in a hcp-like environment. When $Q_{6}^{\text {thr }}$ is low, the $W_{4}$ probability distribution is almost symmetrical, indicating that there is little preference between the fcc-like and hcp-like environments. As $Q_{6}^{\text {thr }}$ is increased, the $W_{4}$ probability distribution become more and more peaked towards the region of $W_{4}<0$. Such an observation suggests that the liquid particles with high enough $Q_{6}$ have a clear preference for the fcc-like symmetry over the hcp-like one. The middle panel of Fig. 2 shows the $W_{6}$ probability distribution for precursor particles with different $Q_{6}^{\text {thr }}$. When increasing $Q_{6}^{\text {thr }}$, the peak of the curve only moves a little bit towards a negative value of $W_{6}$ which characterize both fcc and hcp symmetries. This suggests that the total number of both fcc-like and hcp-like precursor particles is slightly larger than that of 

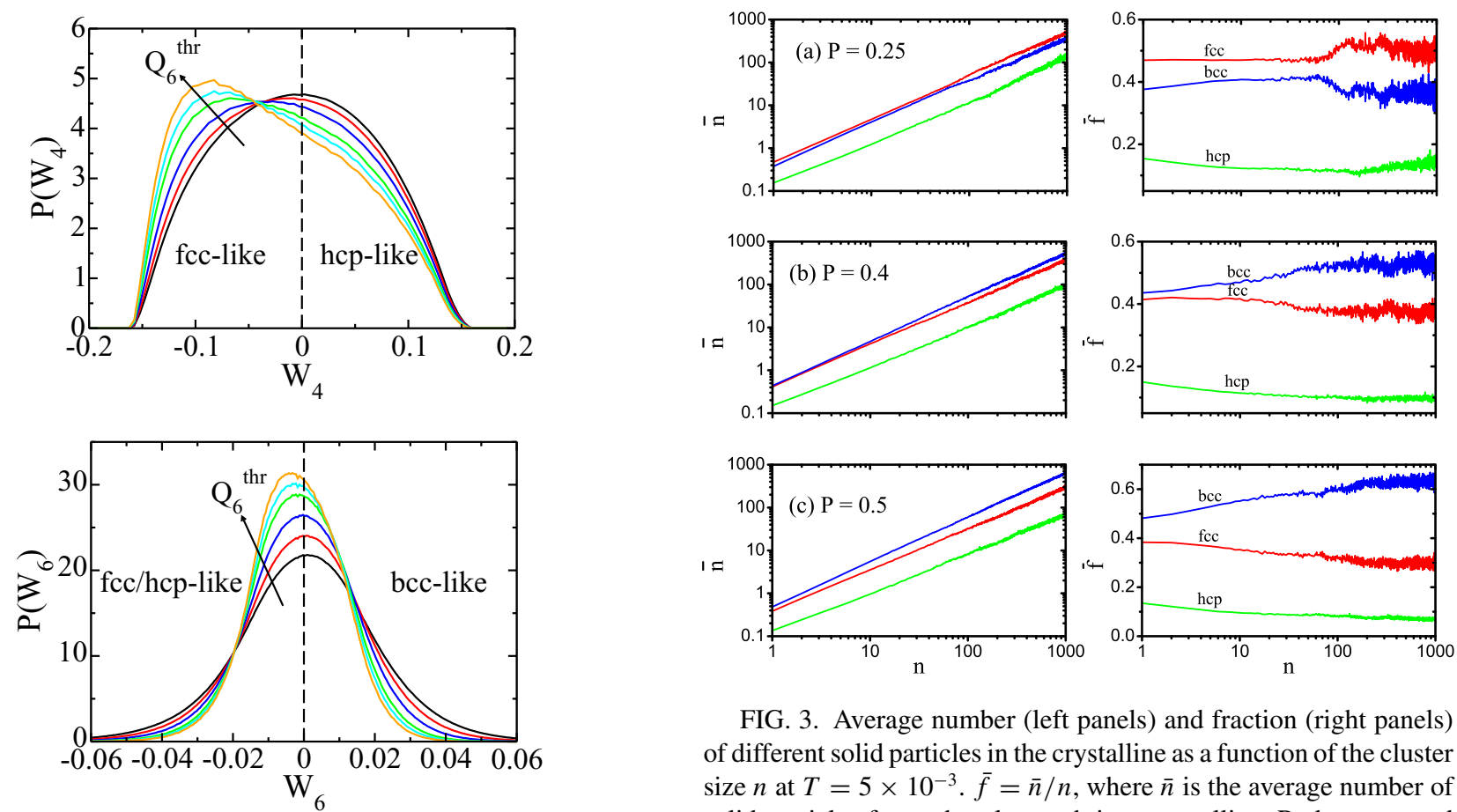

FIG. 3. Average number (left panels) and fraction (right panels) of different solid particles in the crystalline as a function of the cluster size $n$ at $T=5 \times 10^{-3} \cdot \bar{f}=\bar{n} / n$, where $\bar{n}$ is the average number of solid particles for each polymorph in a crystalline. Red, green, and blue lines represent the average number or fraction of fcc, hcp, and bcc particles, respectively. (a) $P=0.25$. (b) $P=0.4$. (c) $P=0.5$.

point $T=5 \times 10^{-3}$ and $P=0.25$. Obviously, fcc particles are dominant over hcp and bcc particles during the nucleation and growth event. This is rather different from the previous observations of other core-softened systems. For instance, Russo and Tanaka investigated the homogeneous nucleation of a GCM system and found that the bcc phase was always favored, despite the underlying phase diagram [32]. As seen in Table I, the free energy of the bcc crystal is closest to that of the fluid phase. Therefore, the bcc phase should be dominant if Ostwald's step rule holds true, but it is not the case as far as that is concerned.

In the top panel of Fig. 4, the number of crystal particles for each polymorph during a typical crystallization process is presented. After a metastable stage, the nucleation and crystal growth starts, causing the number of solid particles to increase until the whole system is almost completely crystallized (see the final configuration displayed in the bottom panel of Fig. 4). During the growth of the nucleus, the fcc structure is always favored, consistent with the observation of Fig. 3(a). Figure 5 shows some snapshots during the crystallization process. Apparently, the nucleation occurs inside of the liquid particles with high $Q_{6}\left(Q_{6} \geqslant 0.3\right)$, which are considered as precursors. In this typical nucleation process, the fcc phase occupies the core of the nucleus attached with very few hcp and bcc particles [see Fig. 5(b)]. When the nucleus grows, it is always embedded in the region of the precursors. In other words, the precursors are wetting on the crystals during the crystal growth.

For a deeper view of the crystallization process, it is also necessary to know the local densities of the particles which are considered to be a good order parameter for translational ordering. Here, the local density of each particle is calculated via Voronoi diagrams, i.e., $\rho_{i}=1 / v_{i}$, where $v_{i}$ is the volume of the polyhedron subdivided for each particle. Figure 6 shows 

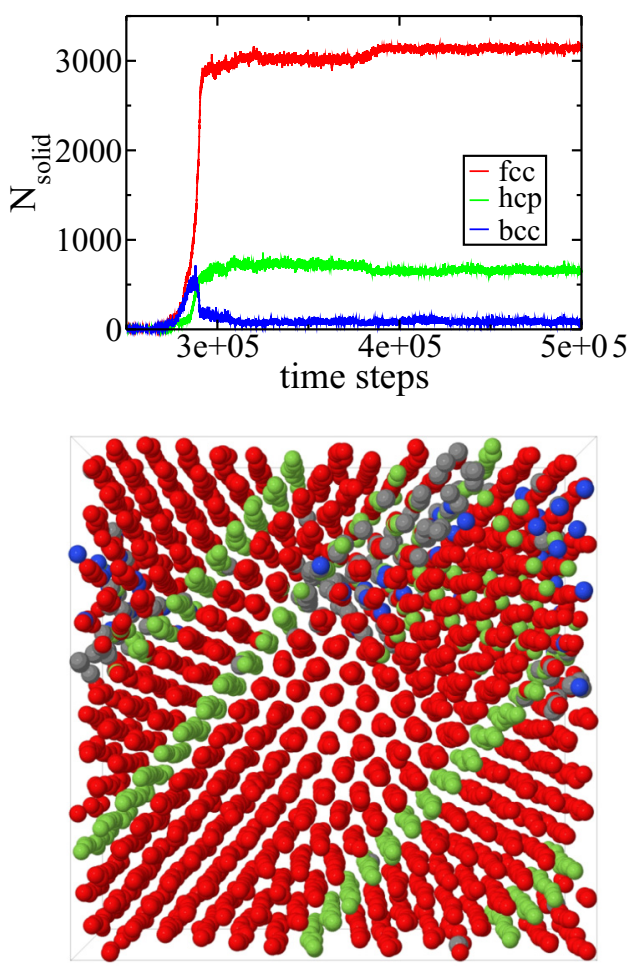

FIG. 4. Top: Evolution of the number of crystal particles for each polymorph during a typical crystallization process. Bottom: The configuration obtained when the crystallization is completed. The state point is $T=5 \times 10^{-3}$ and $P=0.25$.

the relation between the number of solid particles and the average density of each kind of particle (liquid particles with $Q_{6}<0.3$, crystals, and precursors, respectively). It can be clearly seen that the density of the precursors is only a little bit lower than that of the crystals. Such an extremely small density difference between the precursors and crystals suggests that the liquid-solid transition happens microscopically with a very small density change. On the other hand, the densities of both the liquid and crystal particles are not dependent on the crystal size, indicating that the growth of the crystals does not cause any change in the density. Thus, we can conclude that the translation ordering (density) is trivial in the crystallization of Hertzian spheres, but the bond orientational order parameter, here referring to $Q_{6}$, plays a primary role because the liquid-solid transition involves an apparent increase of $Q_{6}$. This point is further confirmed by the maps in the $\left(Q_{6}, \rho\right)$ plane with different colors representing different numbers of connected neighbors $\zeta$ of each particle (see Fig. 7, corresponding to the snapshots shown in Fig. 5). The density change is weakly related to the crystal formation and growth. On the contrary, the development of $Q_{6}$ is strongly correlated with the crystallization process. Before nucleation, most of the liquid particles lie in the region of small $Q_{6}$ while the solid particles lie in the region of large $Q_{6}$. After nucleation starts, more and more particles that originally have small $Q_{6}$ move to the region of large $Q_{6}$, suggesting an increase of crystallinity.

Upon increasing the pressure and keeping the temperature fixed, let us see what will happen. We set the pressure $P=0.4$. The critical pressure of the fcc-bcc phase transition,

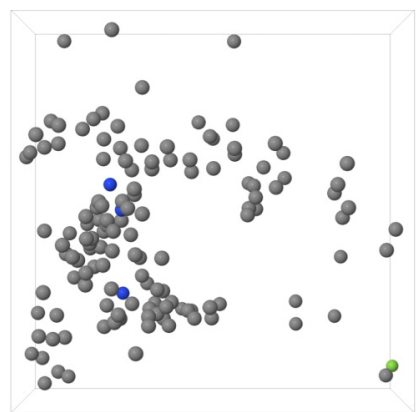

(a)

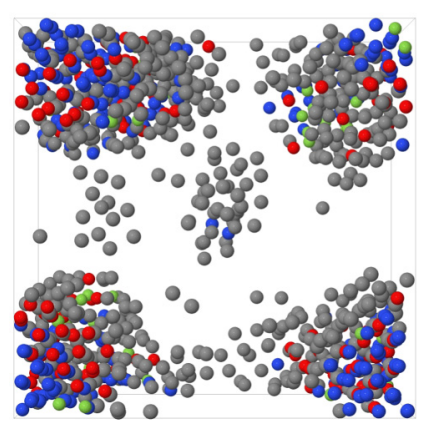

(c)

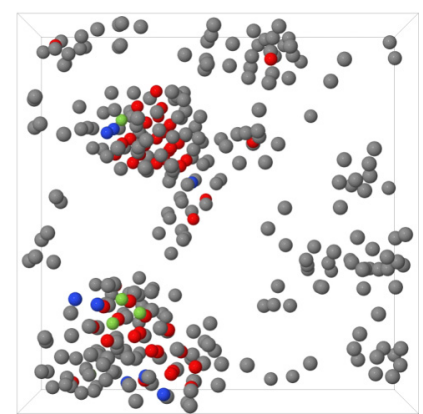

(b)

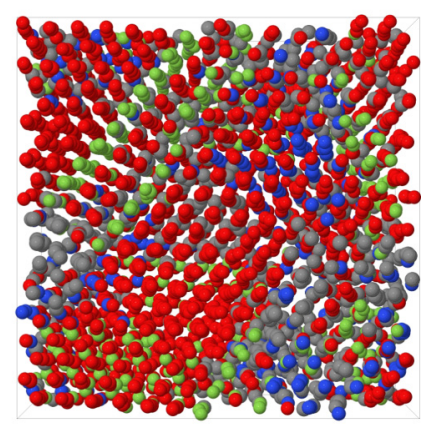

(d)
FIG. 5. Snapshots of a typical crystallization at the state point of $T=5 \times 10^{-3}$ and $P=0.25$. The precursors (liquid particles with $Q_{6} \geqslant 0.3$ ) are depicted with gray spheres. Red, green, and blue spheres represent fcc, hcp, and bcc particles, respectively. (a) $t=2 \times 10^{5} \delta t$. (b) $t=2.7 \times 10^{5} \delta t$. (c) $t=2.8 \times 10^{5} \delta t$. (d) $t=2.9 \times 10^{5} \delta t$.

based on the phase diagram of Hertzian spheres, is $P_{c} \approx 0.44$ at $T=5 \times 10^{-3}$. This means that the pressure $P=0.4$ is close to the boundary of the fcc-bcc phase transition, but the system still has a thermodynamically stable fcc phase. As seen from Fig. 3(b), the average fraction of the bcc phase is dominant over that of the fcc and hcp phases during crystal nucleation. Among these 100 independent simulations, the system is mostly crystallized into a predominantly metastable bcc phase. The tendency for the system to be more likely nucleated into the crystals with a predominant bcc structure seems to be a manifestation of Ostwald's step rule, because

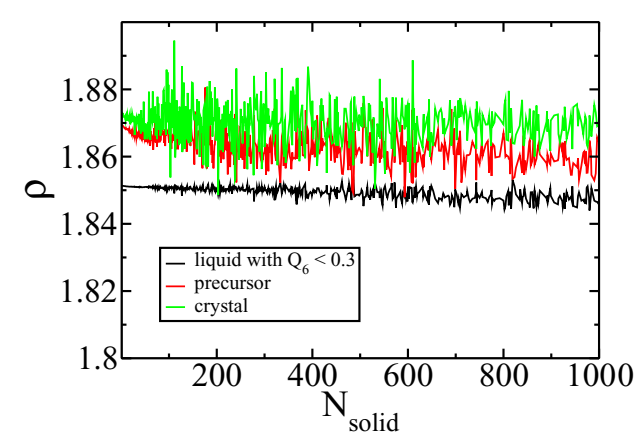

FIG. 6. Relation between the total number of solid particles and the average density for liquid particles with $Q_{6}<0.3$, precursors, and crystals, respectively. The state point is $T=5 \times 10^{-3}$ and $P=0.25$. 

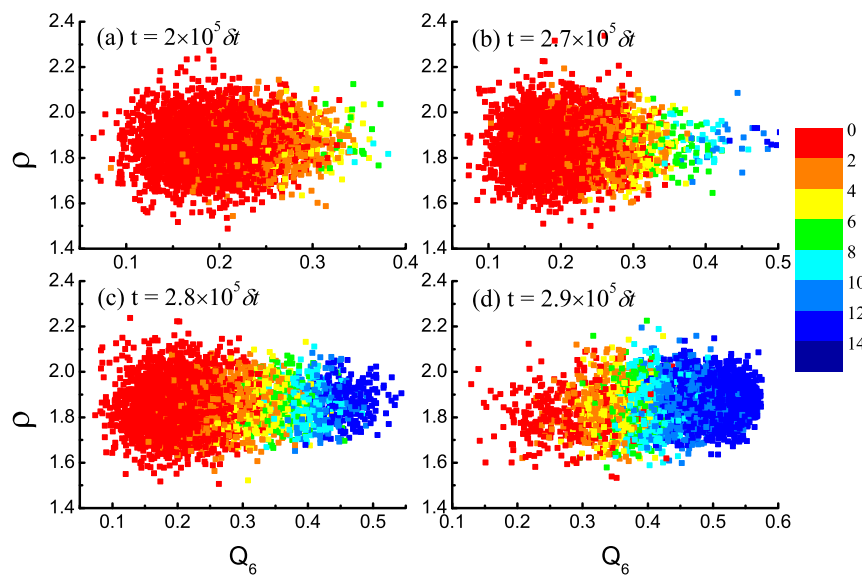

FIG. 7. Temporal evolution of the solid bond number $\zeta$ in the $\left(Q_{6}, \rho\right)$ plane at the state point $T=5 \times 10^{-3}$ and $P=0.25$. $\zeta$ grows from 0 to 14 , represented by the color of each symbol. Notice that a particle with $\zeta \geqslant 8$ is identified as a crystal.

the free energy of the bcc phase is closest to that of the liquid (see Table I). Of course, this phenomenon can also be explained by the mechanism proposed by Alexander and McTague [2], which states that the formation of the bcc phase has a higher probability than the fcc phase at high temperature. When we increase the pressure to the region of a thermodynamically stable bcc phase, e.g., $P=0.5$, the system tends to be crystallized into the structures in which the bcc phase is still dominant [see Fig. 3(c)]. Furthermore, we have also studied the structure of the precursors when the pressure is increased, to speculate that bcc-like particles in the precursors are still dominant despite the crystal structure.

The variation of the pressure seems to make the system change from a hard-sphere-like behavior (low $P$ ) to a softsphere-like behavior (high $P$ ), comparable to the crystallization of spheres modeled with an inverse-power-law potential in which the potential provides a continuous path from hard spheres to the one-component plasma [46]. On the other hand, the effect of $P$ seems to be opposite to the findings in the crystallization of supercooled liquid xenon whose interaction has an attractive part [47]. The observations above, especially the metastable bcc phase near the fcc-bcc boundary, is also reminiscent of recent experimental evidence of a metastable bcc to stable fcc phase transition in charged colloids by in situ monitoring structural changes with the reflection spectrometer during the colloidal crystallization [5], which is explained by Ostwald's step rule [1] and the scenario proposed by Alexander and McTague [2]. The experimental data of colloidal crystallization [5] demonstrate that the metastable bcc structure can only be seen in a narrow volume fraction of colloids close to the boundary of the fcc-bcc phase transition. For the crystal nucleation of Hertzian spheres, we speculate that the region of the pressure where the metastable bcc phase is dominant is exactly in the region of the fcc phase and near the fcc-bcc boundary, which is comparable to the experiments of colloidal crystallization [5]. As is suggested in the scenario of Alexander and McTague [2], nucleation of the bcc phase is uniquely favored under small supercooling in a fluid exhibiting a weak first-order phase transition. Therefore, a question arises
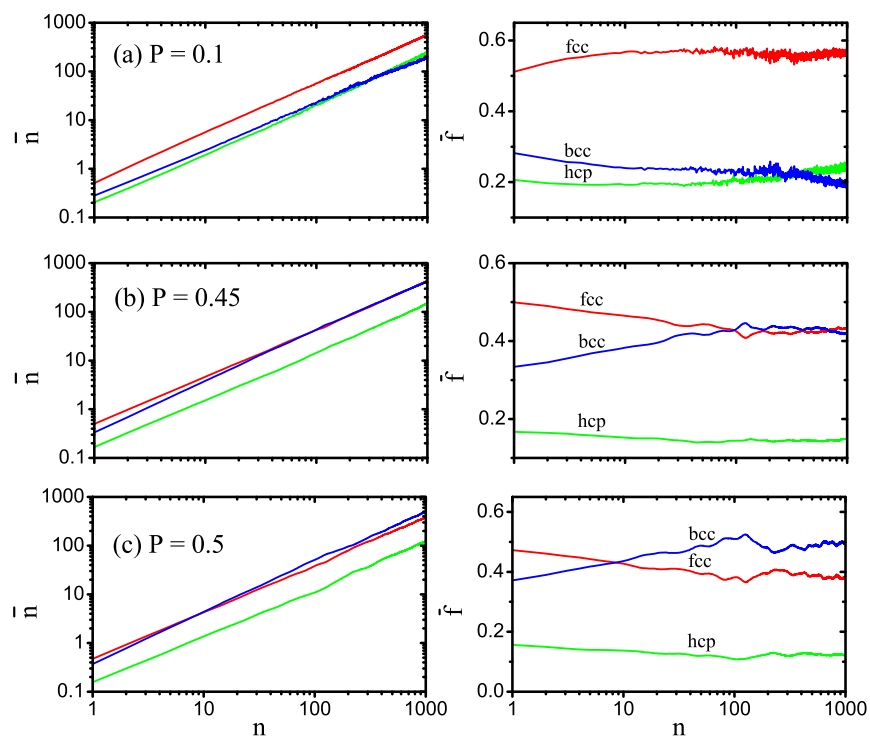

FIG. 8. Average number (left panels) and fraction (right panels) of different solid particles in the crystalline state as a function of the cluster size $n$ at $T=2.5 \times 10^{-3}$. The representation of the lines is the same as that in Fig. 3. (a) $P=0.1$. (b) $P=0.45$. (c) $P=0.5$.

as to whether the criterion can also work for the crystallization of Hertzian spheres under a high degree of supercooling.

Thereafter, we decrease the temperature to far below the melting point, here typically $T=2.5 \times 10^{-3}$, but do not observe any apparent evidence of Ostwald's step rule applying to the crystallization process. Even near the boundary of the fcc-bcc phase transition where fcc is a stable phase, a bcc crystal is not seen to be apparently dominant during nucleation. As far as the pressures are concerned at $T=2.5 \times 10^{-3}$, fcc structures seem to be favored in the small nuclei from $P=0.1$ to $P=0.5$ (see Fig. 8). Notice here that it is not the fcc but the bcc phase that is thermodynamically stable at $P=0.5$. Actually, the bcc structure, until pressure is increased to a high enough amount, becomes dominant in the small nuclei. Moreover, we should mention that the precursors, which appear first before the nucleation and exist through the growth of the nucleus, still have more bcc-like particles than fcc-like and hcp-like particles (see Fig. 9).

In order to further understand the nucleation mechanism, the mean first-passage time (MFPT) method [48,49] is used to extract information about the crystal nucleation. The average time at which the largest nucleus with size $n$ appears for the first time is given by

$$
\tau(n)=\frac{1}{2 J V}\left\{1+\operatorname{erf}\left[c\left(n-n^{*}\right)\right]\right\} .
$$

By fitting the formula above, we can get the critical size of the nucleus $n^{*}$, the nucleation rate $J$, and the Zeldovich factor $Z=$ $c / \sqrt{\pi}$. Here, we should mention that the curve of $\tau(n)$ often does not have a plateau, indicating that the free-energy barrier is relatively low in a direct MD simulation of crystallization. Nonetheless, the fitting parameters in Eq. (12) can still be obtained accurately. As is shown in Fig. 10, the nucleation rate of different structures including $J_{\mathrm{fcc}}, J_{\mathrm{hcp}}$, and $J_{\mathrm{bcc}}$ have been calculated for each state point. In all cases, $J_{\mathrm{hcp}}$ is the 

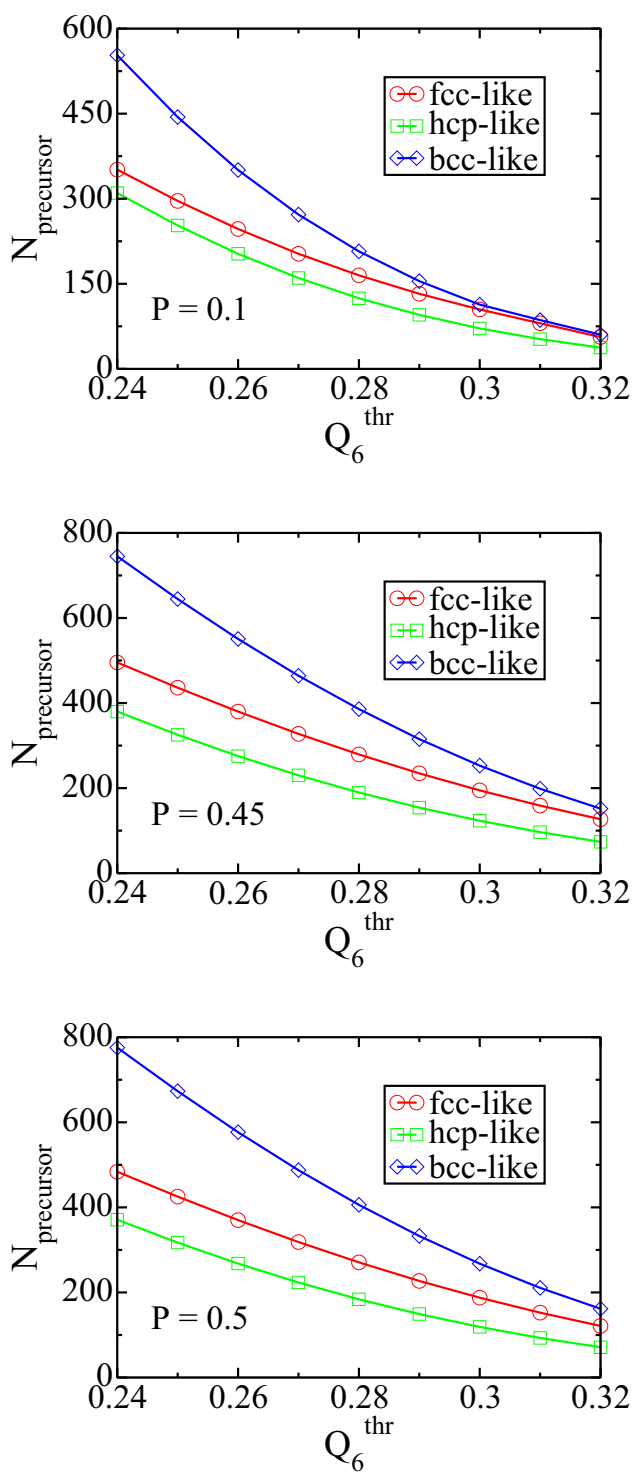

FIG. 9. The number of precursor particles with different $Q_{6}$ threshold $Q_{6}^{\text {thr }}$ in a metastable fluid. The supercooling temperature is $T=2.5 \times 10^{-3}$. Top: $P=0.1$. Middle: $P=0.45$. Bottom: $P=0.5$.

lowest one. At $T=5 \times 10^{-3}, J_{\text {bcc }}$ becomes more and more higher than $J_{\mathrm{fcc}}$, suggesting bcc nucleation is favored with an increase of pressure. At $T=2.5 \times 10^{-3}$, first $J_{\text {fcc }}$ is higher than $J_{\mathrm{bcc}}$ when $P \leqslant 0.45$, and then $J_{\text {fcc }}$ becomes lower than $J_{\mathrm{bcc}}$ when pressure continues to increase. Those observations are actually consistent with the results of the polymorph selection shown in Figs. 3 and 8 . According to CNT, the nucleation rate $J$ is assumed to be $J=\kappa \exp \left(-\Delta G^{*} / k_{B} T\right)$, where $\kappa$ is a kinetic prefactor that is proportional to the self-diffusion coefficient $D_{s}$ of the fluid. We have speculated the self-diffusion coefficient at the fluid state, to find the Hertzian sphere fluid exhibits a clear nonmonotonic behavior ( $D_{s}$ reaches a minimum in the vicinity of $P=0.6$ ) when compressing the system or increasing the pressure. Such dynamical anomalies have also been seen before in Ref. [28]. At $T=0.005$, the nucleation rate increases rapidly to reach a
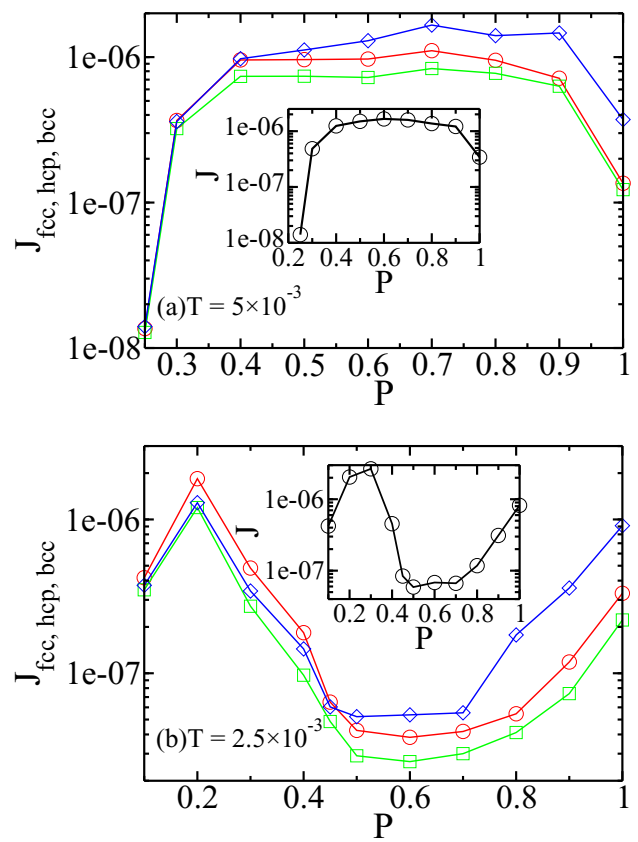

FIG. 10. Nucleation rate calculated by the means of mean firstpassage time (MFPT). Red circles, green squares, and blue diamonds represent the nucleation rate of fcc $\left(J_{\mathrm{fcc}}\right)$, hcp $\left(J_{\mathrm{hcp}}\right)$, and bcc $\left(J_{\mathrm{bcc}}\right)$ particles, respectively. The inner panel displays the nucleation rate $J$ of total solid particles. (a) $T=5 \times 10^{-3}$. (b) $T=2.5 \times 10^{-3}$.

maximum, and then decreases with compression [see the inner panel of Fig. 10(a)]. At $T=2.5 \times 10^{-3}$, the nucleation rate first reaches a maximum at $P=0.3$, subsequently decreasing to a minimum plateau (from $P=0.5$ to $P=0.7$ ), and then increasing again with increasing pressure [see the inner panel of Fig. 10(b)]. Such an observation of $J$ is rather different from or even opposite to the behavior of $D_{s}$, indicating that the role of $D_{s}$ on nucleation is not that significant compared to the free-energy barrier. As seen from Table I, the liquid-bcc interfacial free energy is less than the liquid-fcc interfacial free energy when $P \leqslant 0.45$, which means that the formation of a bcc-liquid interface will cost less energy. Here, the role of the liquid-bcc or liquid-fcc interfacial free energy in the nucleation free-energy barrier can perhaps explain the behavior of the nucleation rate with increasing pressure. At $T=5 \times 10^{-3}$, due to the formation of bcc becoming more and more favorable (see Fig. 3), the free-energy barrier reaches a minimum, resulting in a maximum of $J$. At $T=2.5 \times 10^{-3}$, the fcc structure is always dominant in the nucleus until $P=0.5$ (see Fig. 8), so the free-energy barrier reaches a minimum first when compressing the system from the melting state, and then increasing to a maximum due to the competition between fcc and bec formation.

\section{Discussion}

To establish stable and metastable equilibrium structures, the free energies of the competing fcc, hcp, and bcc structures have been calculated via a thermodynamic integration method for two temperatures $\left(T=5 \times 10^{-3}\right.$ and $\left.T=2.5 \times 10^{-3}\right)$. Compared with the phase diagram published by Pàmies et al. in Ref. [28], however, our results do not fully agree with it. 
Most importantly, as is shown in Table I, the free energy of the hcp structure is always lower than that of the fcc structure at $T=2.5 \times 10^{-3}$, suggesting that the hcp structure should be more stable than the fcc structure. Such results are different from the report of Pàmies et al. in which the fcc phase is the stable one at all temperatures [28].

We have seen that it is not density but the bond orientational order parameter $Q_{6}$ that plays a major role in the crystallization of Hertzian spheres. The nucleation is initiated in precursors which are spontaneously formed in the supercooled state and locally have a specific rotational symmetry (but no translational symmetry). Such a picture is the same as recent observations in both hard spheres [17,18] and soft particles [32], except for some subtle details. In hard spheres, bond orientational order triggers the nucleation process, but the density of the small nucleus appears to be significantly smaller than that of bulk crystals. In the crystallization of soft particles, such as GCM [32] and Hertzian spheres, the density of crystals is not at all dependent on the crystal size.

At first glance, the different nucleation behaviors between high temperatures and low temperatures appear to be related to Ostwald's step rule or the mechanism of Alexander and McTague which is associated with the free-energy barrier of activation. As for the homogeneous nucleation, the free-energy barrier decreases as $1 / \Delta T^{2}$, where $\Delta T$ is the degree of supercooling. Therefore, a low degree of supercooling (a high temperature that is close to the melting point) results in a high free-energy barrier which requires the particles to find an efficient pathway for nucleation. When the temperature is low (a high degree of supercooling), the free-energy barrier is decreased low enough so as to make the effect of Ostwald's step rule or the Alexander-McTague mechanism completely insignificant. From the results of the free-energy calculations (see Table I) and observations of nucleation events in MD simulations, we should stress that Ostwald's step rule is not valid for most of the state points. For a low degree of supercooling $\left(T=5 \times 10^{-3}\right)$, Ostwald's step rule or the Alexander-McTague mechanism seem to be valid only in the vicinity of a fcc-bcc phase boundary.

However, the picture of Ostwald's step rule or the Alexander-McTague mechanism is different from that of precursor mediated crystallization. As recently proposed [17-19], nucleation in precursors is easier due to symmetry matching and the resulting reduction of the interfacial energy. The crystal phase nucleated in the precursors is often the crystal with the highest free energy, as Ostwald's step rule predicts. Thus, the physics behind these two mechanisms (precursor mediated crystallization and Ostwald's step rule) is not equivalent: The former focuses on the interfacial energy, whereas the latter focuses on the bulk free energy. As the seeds of crystallization, the precursors can in principle influence the polymorph crystals. At various state points, we have studied many configurations during the entire crystallization process to find that bec symmetry is always dominant in the precursors. This indicates that the crystal nucleus is wetted by the liquid particles that are predominantly bcc-like ordered. Microscopically, such a preference for bcc symmetry in precursors, comparable to the scenario of Alexander and McTague, can possibly explain the appearance of a high degree of bcc-like order near the fcc-bcc boundary as the mediated precursors favor the formation of bcc crystals.

The effect where the bcc structure is favored in small nuclei is relatively weak (see Fig. 3) compared with earlier observations in other systems with soft-core interactions, such as Lennard-Jones particles [6,7]. This is probably due to the fact that the supercooling used $\left(T=5 \times 10^{-3}\right)$ in our direct MD simulation of crystallization is relatively high so as to make the free-energy barrier relatively low. For a very high degree of supercooling $\left(T=2.5 \times 10^{-3}\right)$, the AlexanderMcTague mechanism no longer works well. Opposite to the case of high temperature $\left(T=5 \times 10^{-3}\right)$ and earlier observations of Lennard-Jones particles [6,7], it is not the bcc structure but the fcc structure that is favored in small nuclei from $P=0.1$ to $P=0.5$ (see Fig. 8). Especially, the dominant structure even changes from fcc to bcc at $P=0.5$, where bcc is thermodynamically stable. This interesting phenomenon, we should stress, is rather unexpected. On the other hand, the small solid particles that are from precursors with a preference for bcc symmetry (see Fig. 9) become predominantly fcc, so we can say that the symmetry of precursors cannot determine the polymorph selection of small nuclei, at least for low enough temperatures.

\section{CONCLUSION}

Molecular dynamics simulations in an NPT ensemble are performed to investigate the crystallization process of a core-softened model system, i.e., Hertzian spheres. Our simulation results show that the polymorph selections and nucleation pathways of Hertzian spheres are rather complex. In NPT simulations, we used two typical temperatures below the melting point that represent different supercoolings, and varied the pressures based on a previously constructed phase diagram. At low pressures, the particles can avoid overlap easily and there are few particles within their diameters, with the result that Hertzian spheres share many similarities with hard spheres. Therefore, the system tends to be crystallized into crystal structures in which the fcc phase is dominant over hcp and bcc phases. As the pressure is increased to make the system compressed, the particles have more and more overlaps and bear more characteristics of soft particles, making the crystallization process complex at different temperatures. At high temperature that represents a low degree of supercooling, the fraction of bcc structures over fcc structures is slightly enhanced so that the system is likely to be nucleated into a crystalline system with predominant bcc ordering in the region of the fcc phase near the fcc-bcc boundary, which can be regarded as validity of the Alexander-McTague mechanism [2]. At low temperatures that represent a high degree of supercooling, in contrast, the Alexander-McTague mechanism is not valid and the small nuclei favor the fcc structure, and the dominant structure even changes from fcc to bcc at $P=0.5$ where bcc is the most stable phase.

By analyzing many trajectories in both supercooled fluid and the crystallization stage, we have seen that the difference in the average density between solid and liquid particles is extremely small, suggesting that translational ordering (density) is not the key player. Another finding about the 
density is that the average density of crystals always remains constant as the crystals grow, which was also observed in GCM [32]. The bond orientational ordering play a major role as its development drives the nucleation and the growth of the nucleus. We also speculate that preordered precursors who act as the seed have structures where bcc-like particles are always dominant despite different state points. The crystallization behaviors mentioned above are probably common to all the soft particles, which calls for further studies in a variety of other systems for confirmation.

\section{ACKNOWLEDGMENTS}

This work is supported by Grants No. 11572322, No. 11172302, and No. 11032011 from the National Natural Science Foundation of China.
[1] W. Ostwald, Z. Phys. Chem. 22, 289 (1897).

[2] S. Alexander and J. McTague, Phys. Rev. Lett. 41, 702 (1978).

[3] C. Notthoff, B. Feuerbacher, H. Franz, D. M. Herlach, and D. Holland-Moritz, Phys. Rev. Lett. 86, 1038 (2001).

[4] J. Bang and T. P. Lodge, Phys. Rev. Lett. 93, 245701 (2004).

[5] S. H. Xu, H. W. Zhou, Z. W. Sun, and J. C. Xie, Phys. Rev. E 82, 010401 (2010).

[6] P. R. ten Wolde, M. J. Ruiz-Montero, and D. Frenkel, Phys. Rev. Lett. 75, 2714 (1995).

[7] P. R. tenWolde, M. J. Ruiz-Montero, and D. Frenkel, J. Chem. Phys. 104, 9932 (1996).

[8] C. Desgranges and J. Delhommelle, Phys. Rev. Lett. 98, 235502 (2007).

[9] J. Delhommelle, Mol. Simul. 37, 613 (2011).

[10] J. F. Lutsko and G. Nicolis, Phys. Rev. Lett. 96, 046102 (2006).

[11] H. J. Schöpe, G. Bryant, and W. van Megen, Phys. Rev. Lett. 96, 175701 (2006).

[12] T. H. Zhang and X. Y. Liu, J. Am. Chem. Soc. 129, 13520 (2007).

[13] J. R. Savage and A. D. Dinsmore, Phys. Rev. Lett. 102, 198302 (2009).

[14] T. Schilling, H. J. Schöpe, M. Oettel, G. Opletal, and I. Snook, Phys. Rev. Lett. 105, 025701 (2010).

[15] G. I. Tóth, T. Pusztai, G. Tegze, G. Tóth, and L. Gránásy, Phys. Rev. Lett. 107, 175702 (2011).

[16] C. Valeriani, E. Sanz, P. N. Pusey, W. C. K. Poon, M. E. Cates, and E. Zaccarelli, Soft Matter 8, 4960 (2012).

[17] T. Kawasaki and H. Tanaka, Proc. Natl. Acad. Sci. USA 107, 14036 (2010).

[18] J. Russo and H. Tanaka, Sci. Rep. 2, 505 (2012).

[19] H. Tanaka, Eur. Phys. J. E 35, 113 (2012).

[20] T. S. Li, D. Donadio, L. M. Ghiringhelli, and G. Galli, Nat. Mater. 8, 726 (2009).

[21] C. Desgranges and J. Delhommelle, J. Am. Chem. Soc. 133, 2872 (2011).

[22] L. Y. Gu, S. H. Xu, Z. W. Sun, and J. T. Wang, J. Colloid Interface Sci. 350, 409 (2010).

[23] C. Desgranges and J. Delhommelle, J. Am. Chem. Soc. 128, 15104 (2006).

[24] W. Z. Ouyang, H. W. Zhou, S. H. Xu, and Z. W. Sun, Colloids Surf. A 441, 598 (2014).

[25] P. Tan, N. Xu, and L. Xu, Nat. Phys. 10, 73 (2014).
[26] C. N. Likos, A. Lang, M. Watzlawek, and H. Löwen, Phys. Rev. E 63, 031206 (2001).

[27] S. Prestipino, F. Saija, and P. V. Giaquinta, Phys. Rev. E 71, 050102(R) (2005).

[28] J. C. Pàmies, A. Cacciuto, and D. Frenkel, J. Chem. Phys. 131, 044514 (2009).

[29] Y. L. Zhu and Z. Y. Lu, J. Chem. Phys. 134, 044903 (2011).

[30] G. Malescio, J. Phys.: Condens. Matter 19, 073101 (2007).

[31] W. Lechner, C. Dellago, and P. G. Bolhuis, Phys. Rev. Lett. 106, 085701 (2011)

[32] J. Russo and H. Tanaka, Soft Matter 8, 4206 (2012).

[33] B. Sun, Z. W. Sun, W. Z. Ouyang, and S. H. Xu, J. Chem. Phys. 140, 134904 (2014).

[34] J. P. Mithen, A. J. Callison, and R. P. Sear, J. Chem. Phys. 142, 224505 (2015).

[35] Z. X. Zhang, N. Xu, D. T. N. Chen, P. Yunker, A. M. Alsayed, K. B. Aptowicz, P. Habdas, A. J. Liu, S. R. Nagel, and A. G. Yodh, Nature (London) 459, 230 (2009).

[36] D. Paloli, P. S. Mohanty, J. J. Crassous, E. Zaccarelli, and P. Schurtenberger, Soft Matter 9, 3000 (2013).

[37] H. G. Wang, X. B. Wu, Z. G. Zhu, C. S. Liu, and Z. X. Zhang, J. Chem. Phys. 140, 024908 (2014).

[38] P. S. Mohanty, D. Paloli, J. J. Crassous, E. Zaccarelli, and P. Schurtenberger, J. Chem. Phys. 140, 094901 (2014).

[39] M. P. Allen and D. J. Tildesley, Computer Simulation of Liquids (Clarendon, Oxford, U.K., 1987).

[40] D. Frenkel and B. Smit, Understanding Molecular Simulation: From Algorithms to Applications, 2nd ed. (Academic, San Diego, 2001).

[41] H. J. C. Berendsen, J. P. M. Postma, W. F. Vangunsteren, A. Dinola, and J. R. Haak, J. Chem. Phys. 81, 3684 (1984).

[42] P. J. Steinhardt, D. R. Nelson, and M. Ronchetti, Phys. Rev. B 28, 784 (1983).

[43] W. Lechner and C. Dellago, J. Chem. Phys. 129, 114707 (2008).

[44] C. P. Royall and S. R. Williams, Phys. Rep. 560, 1 (2015).

[45] M. Leocmach and H. Tanaka, Nat. Commun. 3, 974 (2012).

[46] C. Desgranges and J. Delhommelle, J. Phys. Chem. B 111, 12257 (2007).

[47] C. Desgranges and J. Delhommelle, Phys. Rev. B 77, 054201 (2008).

[48] J. Wedekind, R. Strey, and D. Reguera, J. Chem. Phys. 126, 134103 (2007).

[49] S. E. M. Lundrigan and I. Saika-Voivod, J. Chem. Phys. 131, 104503 (2009). 